\title{
RESONANT RELAXATION
}

\section{SCOTT TREMAINE \\ Princeton University Observatory, Princeton, NJ 08544; E-mail:tremaine@astro.princeton.edu}

The two main arenas of astrophysical dynamics are celestial mechanics and stellar dynamics. The former deals with the motion of few bodies in a near-Kepler potential; the latter with the motion of many bodies in a non-Kepler potential. I would like to discuss the hybrid problem of many bodies in a near-Kepler potential, which is relevant to a number of astrophysical systems, including protoplanetary disks and the centers of galaxies containing massive black holes.

Consider a spherical system of radius $r$, containing $N$ bodies of mass $m$ orbiting in the gravitational field of a body of mass $M \gg N m$. Assume that the orbits have moderate eccentricities and random orientations and imagine taking a time exposure of the system over several orbits. Each body is then smeared into an approximate Kepler ellipse, which precesses slowly on a timescale $t_{\text {prec }}$. Each ellipse exerts a force on other bodies at comparable radius, $f \sim G m / r^{2}$. The mean force from all the ellipses is $F_{m} \sim N f$. The mean force determines the precession time through the relation $L \sim r F_{m} t_{\text {prec }}$, where $L \sim(G M r)^{1 / 2}$ is the specific angular momentum of an orbit. Thus $t_{\text {prec }} \sim(M / N m) t_{\mathrm{cr}}$, where $t_{\mathrm{cr}} \sim\left(r^{3} / G M\right)^{1 / 2}$ is the crossing time.

There is also a stochastic force $F_{s} \sim N^{1 / 2} f$, which depends on the orientation and shape of individual orbits and hence changes on a timescale $t_{\text {prec. }}$. Thus the angular momentum of an individual star random walks, with steps of duration $t_{\text {prec }}$ during which the angular momentum changes by $\Delta L \sim r F_{s} t_{\text {prec }}$. This random walk leads to relaxation of the angular momentum on a timescale

$$
t_{\text {rel }} \sim t_{\text {prec }}\left(\frac{L}{\Delta L}\right)^{2} \sim t_{\text {cr }} \frac{M}{m}
$$

which, remarkably, is independent of $N$ so long as $N$ is large enough that the forces are stochastic. The resonant relaxation rate $1 / t_{\mathrm{rel}}$ is faster than the usual Chandrasekhar or non-resonant relaxation rate by a factor $M /(N m \ln \Lambda)$ where $\ln \Lambda$ is the usual Coulomb logarithm. In contrast, the resonant relaxation rate for the energy is negligible, since the stochastic forces we have described vary only slowly with time.

A massive body travelling through a system of $N$ lighter bodies experiences dynamical friction, a drag force due to its gravitational wake. Similarly, massive bodies orbiting in a near-Kepler $N$-body system experience resonant friction. Resonant friction can lead to either decay or growth of the orbital eccentricity of the massive body, depending on the mean orbital eccentricity of the light bodies.

Resonant relaxation plays an important role in determining the steady-state star distribution around a massive central black hole in a galaxy or star cluster. 
Resonant relaxation and friction are also present in near-Kepler disks. The most interesting case is a disk whose precession rate is dominated by its own selfgravity (e.g. protoplanetary disks, stellar disks close to a massive black hole, but not planetary rings). Resonant relaxation and friction in thin disks can be understood by the marriage of two classic calculations of mathematical physics: Lagrange's secular perturbation theory, which is taken in the continuum limit, and Landau's integration contour, which is used to handle the singular integrands that arise in this continuum limit. Just as the secular interactions of the planets in the solar system are tightly coupled even though the individual planet masses are small, the bodies in a near-Kepler disk are coupled to the low-order, low-frequency $m=1$ normal modes of the disk even when the disk mass is small. As a result, resonant relaxation rates generally cannot be calculated accurately without solving for the disk normal modes and their frequencies; however, we expect that equation (1) remains correct to order of magnitude. Resonant relaxation affects eccentricities and inclinations (but not semi-major axes) and leads to a Rayleigh distribution of these quantities. Resonant relaxation is likely to dominate over non-resonant relaxation in the late stages of planet formation.

Further discussion is given by Rauch and Tremaine (1996), Rauch and Ingalls (1997), and Tremaine (1998).

\section{References}

Rauch, K. P., and Ingalls, B.: 1997, 'Resonant tidal disruption in galactic nuclei', Mon. Not. Royal Astron. Soc., to be published (astro-ph/9710288).

Rauch, K. P., and Tremaine, S.: 1996, 'Resonant relaxation in stellar systems', New Astronomy, 1, $149-170$

Tremaine, S.: 1996, 'Resonant relaxation in protoplanetary disks', Icarus, to be published 